\title{
A Framework of Safety Training Based on Augmented Reality and Cloud Computing Platform in Mines
}

\author{
Feifei Wang ${ }^{1,2}$, Enji Sun ${ }^{1 *}$, Haoyu Wang ${ }^{1,2}$, Shuangyue Liu $^{2}$ \\ ${ }^{1}$ China Academy of Safety Science and Technology, Beijing, China, 100012 \\ ${ }^{2}$ University of Science and Technology Beijing, Beijing, China, 100083
}

\begin{abstract}
As the harsh environment of mines and complicated production processes may easily cause safety production accidents, safety training becomes one of the important ways to reduce personal injuries and property damages. Safety training and education have formed a set of standardized system in the mining enterprises, however, there are still many problems in the operation process of safety training. In order to solve these issues, Augmented Reality (AR) could be used in the traditional safety training based on the comparative analysis of the differences between traditional training and augmented reality-based training. A novel safety training framework is proposed based on AR and Cloud Computing (CC) platform. This framework chooses marker-based AR technology as the research method combined with CC platform for its multiple functions, large-scale and high reliability. Several modules are designed in this framework, which includes mine basic knowledge, equipment cognitive module, intelligent maintenance module, standardized operation module, remote help module, collaborative work module, capability assessment module as well as other functional modules. This framework also achieves the functions such as user registration and login, role management, safety knowledge production training, practical training and periodic assessment functions based on augmented reality. The basic knowledge of mines module is a comprehensive class which contains all the training classes and the regulations to enhance knowledge standard of workers. The equipment cognitive module aims to enhance the employee's mastery of the device. The standardized operation module and intelligent maintenance module utilizes the marker-based AR to improve the technical proficiency of actual practices and skills. The ability assessment module can judge the worker's quality of his work in mines. The background management module is designed for the system administrators to maintain the system and data on cloud platform. In this safety training system, the equipment profile, operating procedures and other training knowledge can be displayed in a more intuitive form. And the abstract training concepts are visualized and interactive. This safety training system can merge the training with the actual operation to improving training efficiency. In this way, it can break through the limitation of safety training methods, time, location, etc. in the traditional safety training. Moreover, it can increase employees understanding of theoretical knowledge and proficiency in operational skills. The AR and CC based training system can not only be used in mines, but also can be designed according to different needs of the corresponding system. It would have significant influence of practice and application to the universities, enterprises, government departments and other fields.
\end{abstract}

Keywords: augmented reality technology, cloud computing platform, mine safety, interactive system, safety training

\section{Introduction}

The production processes of mines is complex, involving exploration, ventilation, transportation, blasting, excavation and other issues (Liu et al 2007). In these issues, the harsh environment and multitudinous large equipment can easily lead to production safety accidents. Except for production factors, up to 50\%-80\% of industrial accidents are caused by human unsafe behaviors (Xu 2017). Training employees is one of the most important ways to reduce personal injuries as well as property damages. It can standardize their work processes, improve employees' safety awareness and the capacity of handling accident. Safety training in mining enterprises has formed a set of standardized system (Gao and Wang 2016). However there are still many problems due to the restrictions of places, dangers, economic benefits and other factors. The existing training method mainly includes the traditional teaching method, practical method, seminar method, the newly introduced virtual reality training and augmented reality training (Liu et al 2016).

Teaching method and seminar method are all centralized theoretical teaching. Although the operating points of mine work can be passed to employees in these two training methods, a great deal of practice is still necessary to operate flexibly. Practical method means that the experienced employees convey experience to new employees in combination with specific operations. It is limited by the habits or experience of the employees. Virtual reality training is that virtual environment is generated

* Corresponding Author: Enji Sun, Email: enjisun@gmail.com, phone: +86 1084916155

Copyright (C 2018 Canamaple Academia Services, http://press.camdemia.ca

DOI: 10.15273/ijge.2018.03.014 
through virtual reality technology to enable trainees' immersion. Many scholars studied the use of VR in the mining industry, including risk pre-control, accident scene reappearance, self-rescue, emergency rescue, simulation training and employees training (Liu et al 2015 and Hong 2013). To some extent, virtual reality could make up for the lack of the combination of theory and practice in traditional training. However, employees are only immersed in the virtual environment, they are lack of interaction with the real scene. Augmented reality training uses augmented reality technology to combine realistic devices with virtual information based on real-world scenarios. Augmented reality technology has found preliminary applications in many fields including auxiliary maintenance, automobile manufacturing, machinery and tool manufacturing, industrial equipment and power grids. For instance, the German BMW laboratory is conducting research on the mechanical maintenance of car, where AR was used as an auxiliary means (Guo 2017). The employees of European Aeronautic Defense and Space Company completed highdensity cabling on board with the help of augmented reality technology. Augmented reality technology takes into account both the real scenarios and the virtual scenarios, which have great assistance in improving the efficiency of employees training.

After analyzing the traditional training, a novel safety training framework is proposed based on augmented reality (AR) and cloud computing (CC) platform. This paper chooses Marker-based augmented reality (AR) technology as research method of the safety training framework. Several functional modules was designed such as mine basic knowledge, equipment cognitive module, intelligent maintenance module, standardized operation module, remote help module, collaborative work and capability assessment module. These modules achieve learning, maintenance, experts answer, evaluation and other functions. This framework helps employees to conduct efficient and interactive training on the operation site. It not only enhances the employees' theoretical knowledge but also increases their operating experience. The safety training system can be designed to the corresponding system according to different needs. It not only could be used in mines, but also has important practical significance and application value to the work of universities, enterprises, government departments and other fields.

\section{Augmented Reality Technology}

Augmented reality technology is developed on the basis of virtual reality technology. It superposes the virtual information generated by computer technology on the devices in the real environment (Cheng 2012). It can enhance the user's perception of the physical environment and reduce the cost of the real model (Liu et al 2014). Compared to virtual reality technology, it does not need to render all the real environment, which reduces the workload and ensures that the interaction between the employees and the real device. The augmented reality technology has great potential in many fields.
Augmented reality technology includes vision-based augmented reality, geo-location based augmented reality, projector-based augmented reality, and augmented reality based on real-time positioning and map construction technologies. Vision-based augmented reality uses computer vision to obtain real scene information and uses image processing to identify and track the real scene. It is divided into marker-based augmented reality and augmented reality based on natural features. Marker-based augmented reality has the characteristics of simple algorithm, high speed, low requirements for environmental conditions and hardware conditions, and strong scalability. The markers is set on the device in advance to assist in determining the spatial position of the camera and the target. Taking into account the system's requirements for real-time and accuracy, marker-based augmented reality was took as the research method.

Display technology, tracking registration technology, interactive technology are the key technology to support the operation of the augmented reality system. The basic process of augmented reality system is shown in Figure 1.

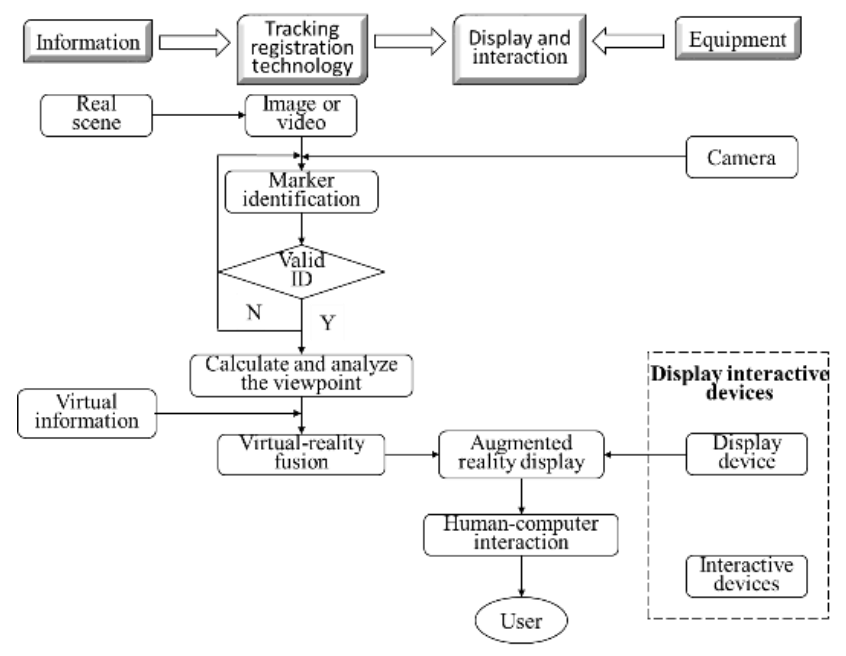

Figure 1 The basic process of augmented reality system

The implementation effects of marker-based augmented reality system is achieved through marker identification, viewpoint calculation, virtual information construction and virtual -reality fusion (Ni et al 2009). Mine employees wear augmented reality device to obtain real scene video by using acquisition equipment. The video is checked to detect the markers in the real scene. If the makers are successfully detected, the related information will be passed to the tracking registration module. This module performs a series of calculations to obtain the spatial location relationship between the users and the devices. Even if the location of the user changes, its spatial relationship with the device can be monitored in real time. According to the detected markers, the virtual information generating module calls the corresponding information to establish the virtual object. The virtual-reality fusion module adds the generated virtual object to the real scene closely on the basis of virtual object and the spatial location 
relationship. The virtual-reality fusion effect is output through the display device.

\subsection{Display technology}

The display technology is the key step to display fusion effects in augmented reality system. The fusion effects of the overlay scenes received by the users is determined by this technology. This technology makes the real scene information and the virtual scene information exist within the uses' visual range simultaneously through the display device and the display algorithm. The display devices include a helmet-mounted display device and a non-helmetmounted display device. The helmet-mounted display device is divided into video see-through display device and optical see-through display device. The former combines the real scene information collected by a helmet-mounted camera and the calculated spatial location of the virtual information to get the virtual-reality fusion scene employees. The latter see the real world and virtual information meanwhile through the optical fusion device, which creates a feeling of combination between them.

The helmet-mounted display device presents a fusion effect between the equipment and auxiliary information needed for normal work. It enables employees to operate flexibly without spending too much energy on the auxiliary information. It liberates employees' hands and greatly facilitates employees training and daily work. The main principle is depicted in the Figure 2.

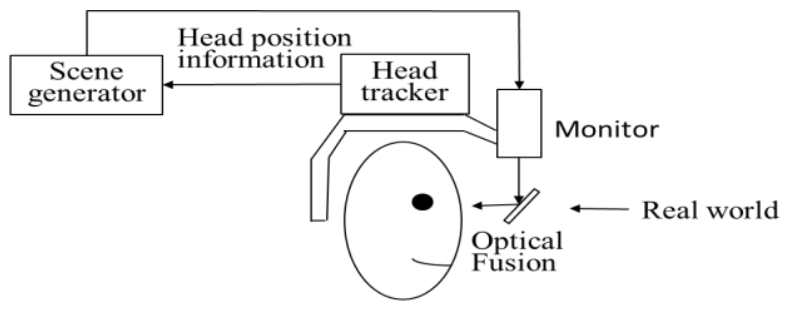

(a) Optical see-through display device

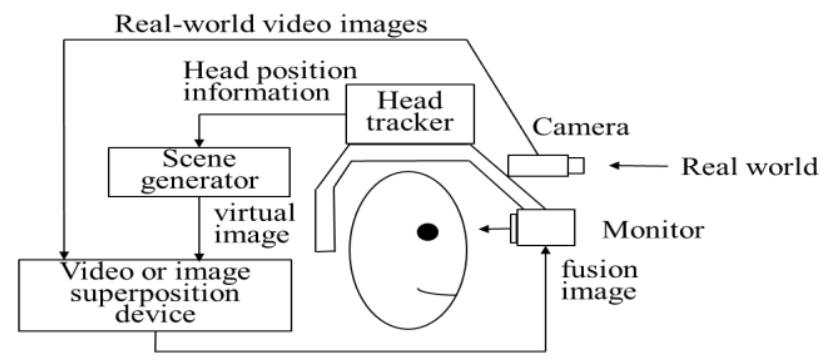

(b) See-through display device

Figure 2 The helmet-mounted display device

\subsection{Tracking registration technology}

Tracking registration technology is one of the core technologies of augmented reality system. It greatly affects the development of augmented reality system. Superimposing the virtual scene information onto the real scene accurately is the main aim of this technology. Even in the case of moving, the seamless connection between the real scene information and the virtual information must be ensured. To achieve this goal, it is necessary to clarify the spatial location of the virtual information in the real environment. This process is registration. However, the spatial location of virtual information is not static. It changes with the user's spatial location or movement status. Tracking mainly focuses on the problem. The spatial location of the virtual information makes corresponding changes with viewpoint. If the tracking system cannot accurately track the user's viewpoint in real time, great errors will be caused by the augmented reality system when the actual situation and virtual information is merged.

The implementation effects are achieved through the conversion among different coordinate systems such as real scene coordinate system, camera coordinate system and the actual image (virtual information) coordinate system in marker-based tracking registration. The relationship among the three coordinate systems as shown in Figure 3.

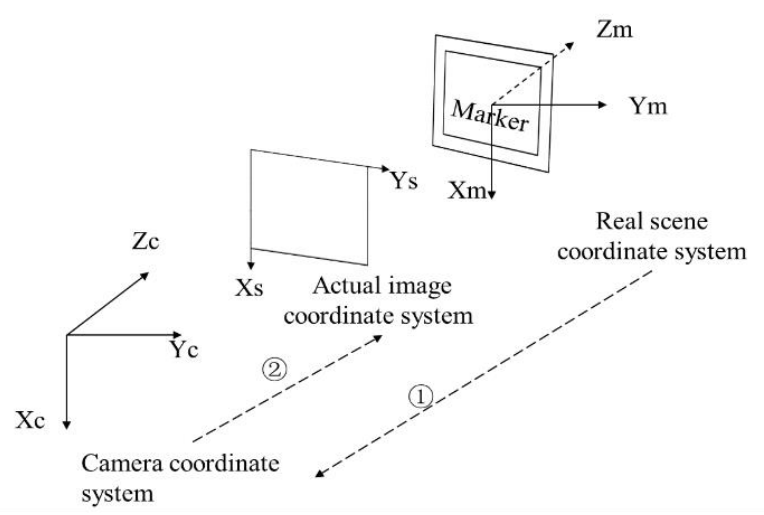

Figure 3 The conversion of tracking registered coordinate system

In order to obtain the accurate spatial location of virtual information, it mainly passes through the three stages of real scene-camera-virtual information. Augmented reality system transforms the real scene coordinate system into the camera coordinate system to get the user's viewpoint information. This process defines the relative position and direction between the real scene coordinate system and the camera. In the next place, the AR system will calculate the conversion relationship between the camera coordinate system and the actual image coordinate system. For the same point $\mathrm{N}$, its coordinates in the real scene coordinate system, camera coordinate system and the actual image coordinate system is $\left(\mathrm{X}_{\mathrm{m}}, \mathrm{Y}_{\mathrm{m}}, \mathrm{Z}_{\mathrm{m}}\right),\left(\mathrm{X}_{\mathrm{c}}, \mathrm{Y}_{\mathrm{c}}, \mathrm{Z}_{\mathrm{c}}\right)$ and $\left(\mathrm{X}_{\mathrm{s}}, \mathrm{Y}_{\mathrm{s}}\right)$. The relationship among the three coordinate systems can be established as the following formulas.

(1) The conversion relation between the real scene coordinate system and the camera coordinate system can be described by equation (1):

$\left[\begin{array}{l}X_{c} \\ Y_{c} \\ Z_{c}\end{array}\right]=\left[\begin{array}{ll}R & T\end{array}\right]\left[\begin{array}{l}X_{m} \\ Y_{m} \\ Z_{m}\end{array}\right]=\left[\begin{array}{llll}r_{1} & r_{2} & r_{3} & t_{1} \\ r_{4} & r_{5} & r_{6} & t_{2} \\ r_{7} & r_{8} & r_{9} & t_{3}\end{array}\right]\left[\begin{array}{c}X_{m} \\ Y_{m} \\ Z_{m} \\ 1\end{array}\right]=W\left[\begin{array}{c}X_{m} \\ Y_{m} \\ Z_{m} \\ 1\end{array}\right]$ 
where, $\mathrm{R}$ is the rotation matrix, $\mathrm{r}_{\mathrm{i}}$ is the rotation parameter, $\mathrm{T}$ is the translation matrix, $\mathrm{t}_{\mathrm{j}}$ is the translation parameters, $\mathrm{W}$ is the total transformation matrix, $X_{c}, Y_{c}, Z_{c}$ are the points in the camera coordinate system, $\mathrm{X}_{\mathrm{m}}, \mathrm{Y}_{\mathrm{m}}, \mathrm{Z}_{\mathrm{m}}$ are the points in the real scene coordinate system

(2) The conversion relation between the camera coordinate system and the actual image coordinate system can be expressed as equation (2):

$$
h\left[\begin{array}{c}
X_{S} \\
Y_{S} \\
1
\end{array}\right]=\left[\begin{array}{ccc}
S_{x} f & k & x_{0} \\
0 & S_{y} f & y_{0} \\
0 & 0 & 1
\end{array}\right]\left[\begin{array}{l}
X_{c} \\
Y_{c} \\
Z_{c}
\end{array}\right]=P\left[\begin{array}{l}
X_{c} \\
Y_{c} \\
Z_{c}
\end{array}\right]
$$

where, $\mathrm{h}$ is the scale factor, $\mathrm{f}$ is the camera focal length, in millimeters, $x_{0}$ and $y_{0}$ are the camera center coordinates in pixels, $S_{x}$ and $S_{y}$ are the $X$-axis, $Y$-axis focal length scaling factor (pixel $/ \mathrm{mm}$ ), $\mathrm{k}$ is the distortion parameter, on behalf of the camera center offset, $\mathrm{P}$ is the camera internal parameter matrix. $X_{c}, Y_{c}, Z_{c}$ are the points in the camera coordinate system, $X_{s}$ and $Y_{s}$ are the points in the actual image coordinate system.

(3) Combined equations (1) and (2), the conversion relation between the real scene coordinate system and the actual image coordinate system can be expressed as equation (3):

$$
h\left[\begin{array}{l}
X_{s} \\
Y_{S} \\
1
\end{array}\right]=P\left[\begin{array}{l}
X_{c} \\
Y_{c} \\
Z_{c}
\end{array}\right]=P W\left[\begin{array}{l}
X_{m} \\
Y_{m} \\
Z_{m}
\end{array}\right]
$$

\subsection{Interactive technology}

Improving the efficiency of work is the main purpose of interactive technology. This technology is an indispensable part of augmented reality system. The users issued commands. The interaction system obtains the instructions issued by the users and gives corresponding feedback after analysis and calculation based on related commands registered in advance (). In general, the choice of interaction mode depends on the nature of the works and the needs of the employees. Because of the complex production environment and large-scale equipment such as scraper, tunneling trolley and more in mining enterprises. The maneuvers are more agility and comfortable by operating with both hands. Interaction mode should not take up too much energy of employees in the process of the equipment operation. In view of this situation, voice interaction only requires employees to use the designated voice to interact with the system naturally. It is a great liberation of employees hands and more suitable for mine employees. The process of voice interaction system is shown in Figure 4.

From the time that the mine employees issue a command to the time the execution of the order, identifying the commands spoken by the employees is a crucial aspect of the voice interaction. Audio from the employees is converted into electrical signals. The electrical signals are sent to the recognition system. The pre-processing (such as noise reduction) is performed firstly to reduce the interference to subsequent steps. The processed electrical signal is framed by moving the window function and the audio features. The audio features are searched and matched with pre-stored acoustic models and language models to find the best match. Then, the commands get triggered to achieve the interaction.

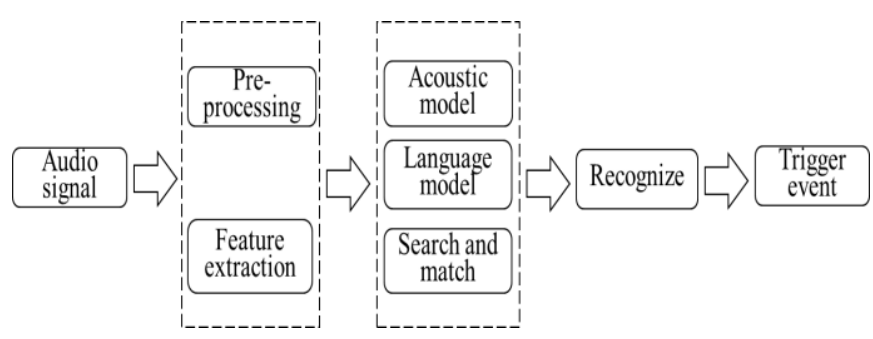

Figure 4 Voice interaction process

\section{Cloud Platform}

The resources required for mine employees training depend on the work area and types of employees. The management of resources is onerous as the complexity of mine production, especially the resources required for employees training are numerous. In order to solve this problem, the cloud platform was used in mine employees training (Sun et al 2012). Cloud platform is a form of cloud computing. Cloud computing is a super-computing model. It can integrate large-scale and scalable computing, storage, data, applications and other distributed computing resources to enable them to work together. The deployment of these server resources is carried out through the cloud platform ( $\mathrm{Li}$ et al 2014). The advantages of introducing a cloud platform in mine training are as following.

(1) Massive scale

Mine enterprises have a large number of employees. Training multiple people simultaneously has high requirements on resources and system. The cloud platform could set up matching servers according to the scale of the mining enterprises. Through the management server, the massive data required for employee training can be processed and stored. Employees can train in system at the same time.

(2) High availability

Cloud platform has a wide range of applications. It supports the use of different enterprises and meets the diverse needs of users at the same time. The cloud platform can store training resources such as relevant laws, regulations, standardization tutorials, employee types, regionalization and characteristics of mines, etc. in multiple storage servers through distributed storage technologies. The application of cloud platform in mining enterprises helps to reduce the economic cost as well as improve resource utilization and system reliability.

(3) Stability

The safety training system of mining enterprises involves huge employees training data and the processing and analysis of these data. Data is of great importance to both companies and individuals. If the system goes wrong, it will cause serious consequences in the progress of training or some other areas. For example, system errors may result in the loss of data when employees are training basic 
knowledge of mines. During the actual operation training, the system is in error, the employees in training may get personal injury or meet death accident if he is unskilled or even does not know the next operation step. In view of this situation, the cloud platform adopts the multi-copy fault tolerance technology, which is greatly helpful for fault tolerance and recovery of data. The cloud platform has dedicated personnel to ensure data security and be not vulnerable to hacking and improve training safety.

\section{(4) Elasticity}

The resources of mining enterprises are dynamic process. The resources, such as regulations and equipment conditions of mine enterprises, will change to some extent when the equipment, employees' numbers and job information of mining enterprises change. For example, the system will add the relevant training materials, equipment profiles when new mine equipment was introduced. It can be seen that the system must be flexible to meet the dynamic needs of enterprises.

\section{System Framework Design}

\subsection{Overall framework}

In order to improve the efficiency of employees training in mining enterprises and reduce the risk of production, a novel cloud computing framework is proposed based on augmented reality. It includes user layer, data layer and application layer. The data layer is the foundation of application layer, and they work together to provide users with service. The relationship among these three parts is shown in Figure 5.

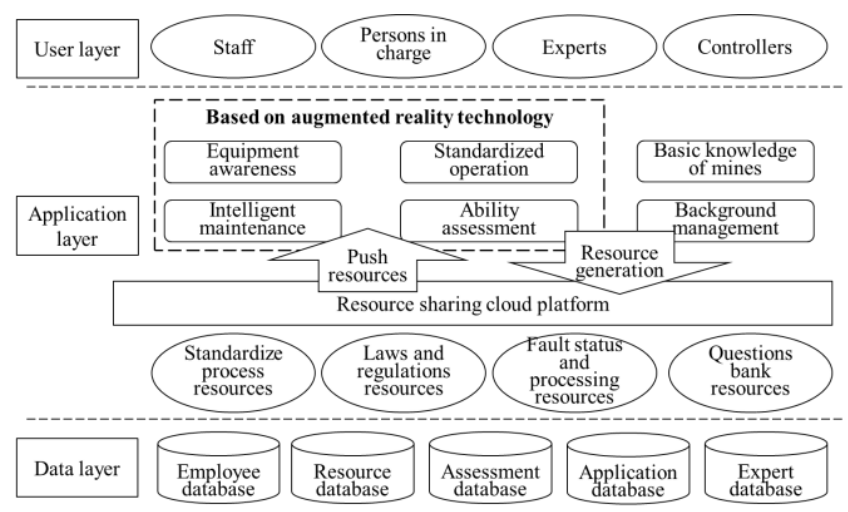

Figure 5 The framework of training in mine

Data layer includes employee database, resource database, assessment database, application database and expert database. The employee database mainly includes the information of mining employees, such as mine general employees, special operators, team leaders, safety managers, system administrators and mine managers. In particular, the certificate information of special operators must be recorded in system. The expert database involves the information of various experts and classifies these experts according to the field that they are good at. Resource database is a core part of the safety training system. It involves a variety of information required for employees training, such as laws, regulations, the structure and principles of equipment. The assessment database includes the information required for assessment of the mine employees' ability, such as the exam bank and the evaluation criteria. The application database is mainly applied to record employee's work and the information needed for actual work. These data stored in the data layer are the basis of the various modules to presentation in the application layer. The application layer sets up training modules such as mine basic knowledge, equipment cognition, intelligent maintenance and standardized operation to improve the ability of mine workers in tunneling, blasting, transportation and overhaul etc. Also it sets up a special ability assessment module to conduct performance assessment. The system is mainly provided to users including employees, experts, persons in charge, controllers and so on. Users call the resources needed to conduct different project training through the cloud platform, especially these four modules based on augmented reality have great help in improving the work ability of the employees. Meanwhile, the resulting resources are saved to the database in the opposite path.

\subsection{Function module}

\subsubsection{The training models based on theory}

The basic knowledge of mines module pre-stores the theoretical knowledge required by employees for daily work in the database, including the mine's production environment, regional division, the role of each region, equipment in different regions, different work types of mine, standardized processes of production operation, safety regulations need to be comply with by miners and other aspects of the theoretical knowledge in mine. In the database, users call or download these resources through the cloud platform for training and learning. The module helps employees grasp the theoretical knowledge, which lays a solid foundation for employees to work in mines.

\subsubsection{The training models based on site}

Equipment awareness, intelligent maintenance, standardized operation combineS theoretical knowledge with equipment at the production site through augmented reality technology. And they use cloud platform as a training tool to present the effect of augmented reality, which have improved the form of purely theoretical teaching in traditional training. Instead. The specific process is shown in Figure 6.

According to the training modules and equipment selected by the employees, the system searches relevant data from the database and performs certain processing to form the auxiliary information needed for the training of employees. These information are formed into a virtual list in the physical operation process or pushed to users in various forms, such as video, audio, images, text and other forms, to prompt the user how to conduct the next operation. Augmented reality training provides employees with the training environment in which virtual information guidance and physical operations are synchronized. It ensures that auxiliary information changes with the position of the 
person in real scene, making the real scene and the virtual information within the range of sight seamlessly connect. The system gives the corresponding response according to different orders. The AR training module such as device recognition, standardized operation, and intelligent maintenance will be described in detail below.

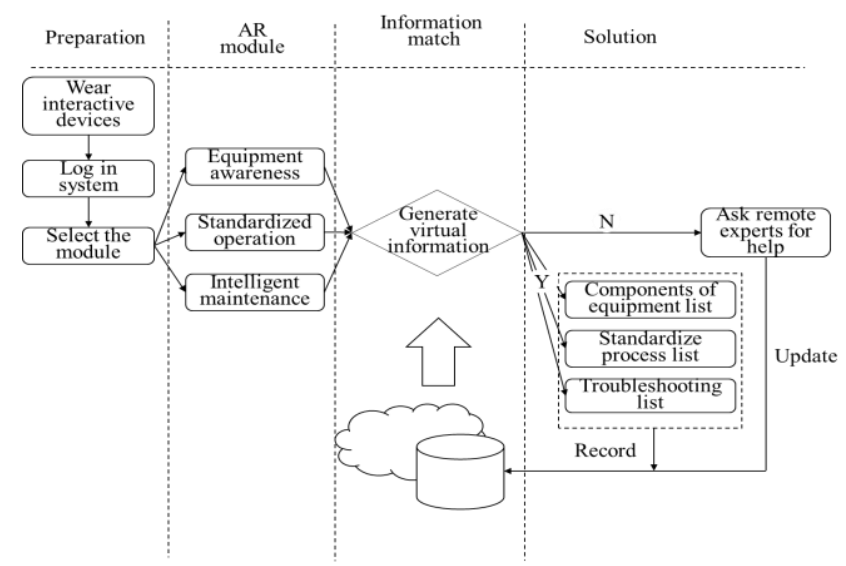

Figure 6 The training process based on field equipment

(1) Equipment cognitive module

The common preconditions for employees of different works is to understand the composition of the equipment involved in the work and the functions of each part. The production facilities in mines are numerous, the production equipment operated by different posts and the standards they need to follow are different. Therefore, it is necessary to carry out targeted training according to the actual situation of various employees. The main training process is shown in Figure 7.

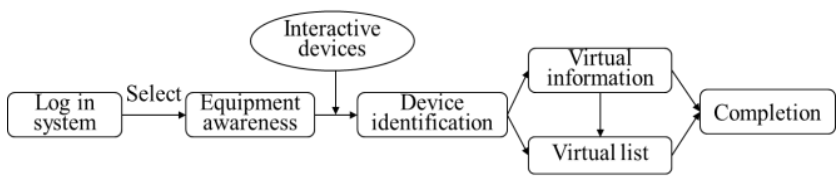

Figure 7 The process of equipment awareness training

After logging in the training safety system and choose the equipment cognitive module, the employees scan the equipment need to be recognized through the interactive equipment worn. This system identifies device, calls the corresponding virtual information and superimposes the virtual information on the equipment through the tracking registration technology. Meanwhile, it generates the virtual list of all the parts of the device. Each completed item can be annotated and recorded in the list by means of voice interaction. Under the module, employees can view the three-dimensional model of devices, understand the functions of various operation buttons of the device, disassemble the device and view the internal structure by using gestures such as dragging. This module expresses the theoretical knowledge in traditional training in an easier-tounderstand manner, which helps employees understand and remember theoretical knowledge. It is suitable for pre-job training for employees and lays a solid foundation for formal work.

(2) Standardized operation module

At present, the on-site employees training is mostly conducted under the leadership of experienced veteran employees, involving the field and operation internship. Subject to the experience or habits of old employees, some of teach contents are wrong, even some are contrary to the standard. All these can lead to safety accidents. In order to reduce the safety risks caused by non-standard operation, it is imperative to standardize the operation of employees. For those who are about to enter the mining enterprise, standardized training will help them to develop their safety awareness and standard behavior. For employees who have been working for several years, standardization can correct their erroneous behavior. In short, standardized training has a wide range of applications. The training of standardized operation is shown in Figure 8.

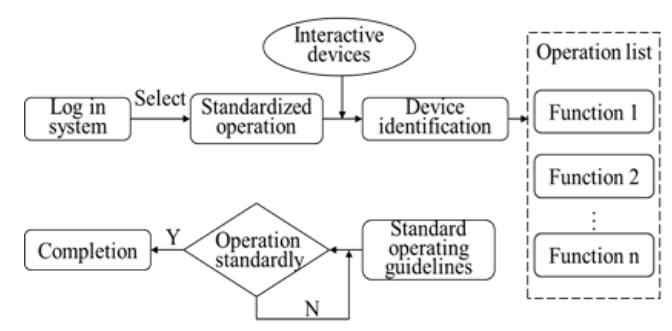

Figure 8 Standardized operation training process

When the employee enters the standardized operation module training, the system identifies device and generates a list of operational functions that the device can perform according to the equipment type. Then the employees select one project to complete by voice interaction. For example, the vehicle's reversing function, forward function during transport. The system generates standardized operation guidance steps according to the project selected by the employees, and verifies the correct operation of each step of the employees. If successful, the next step is taken. Otherwise, the error step is repeated until the operation is completed.

(3)Intelligent maintenance module

There are many reasons that lead to a fault condition. The faults that employees encounter are limited, and some faults exceed the ability of the maintenance workers. In view of this situation, a renewed safety training system with specific steps is particularly important. Maintenance module of the frame includes fault repair and periodic inspection. The training process is shown in Figure 9.

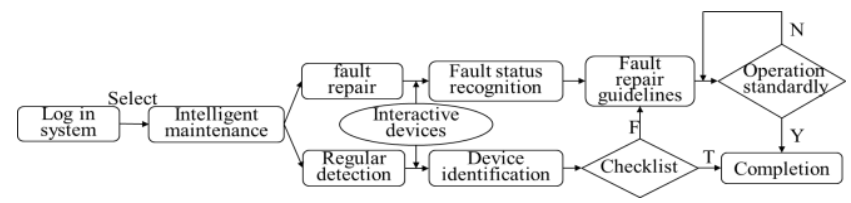

Figure 9 Intelligent maintenance training process 
Periodic inspections are performed by employees to check for weak links and status parameters. Fault repair is the process that employees return the equipment to its normal operating state. According to own needs, the employees choose one from two modes which includes fault repair and periodic inspection. For the fault repair mode, the employees scans the faulted device through the worn interactive device to identify the fault status. The system generates a solution scheme about solving the fault. The employees repairs the equipment according to the fault maintenance guideline. In this process, the system checks whether each step reaches the operation standard. If the standard is reached, the next operation step is performed. If the standard is not reached, the employees repeats this step until the equipment is repaired successfully. For periodic inspection mode, this mode is mainly for the equipment that need regular inspection in mine. The system has the functions of pre-warning the overhaul workers, generating the virtual inspection list and recording the overhaul items. If the fault state is detected during the overhaul, the system will generate corresponding maintenance guidelines to instruct the employees to repair until the fault item completed and recorded.

(4) Remote help module

Equipment awareness, standardized operation, intelligent maintenance are all supported by the resources contained in the database. Due to the complexity of on-site issues, employees are most likely to encounter problems beyond the scope of the system knowledge base. The remote help module is mainly used to solve these problems that go beyond the scope of employees. Employees can ask the experts in remote areas for help through this module. After the employees passing the scenes on site to the expert through the cloud platform, the expert can guide the on-site employees in the form of voice, picture and video. At the same time, the system updates and supplements the database to avoid the same type of problem.

(5) Collaborative work module

Poor communication may lead to unpredictable consequences as the complex mining processes and multiple tasks at once. The collaborative work module is introduced in the system to avoid accidents caused by poor communication. With the development of information technology, employees' behavior can be monitored in real time (Sun et al 2010). When a worker enters a designated area, the system will remind the worker of the hazardous conditions in the area and corresponding precautions. For example, when the blasting work in mines is carried out, the system reminds employees who are going to enter the blasting area in advance by judging the traveling direction of employees. When multiple people carry out a certain work at the same time, they can check the working status of the others and avoid accidents caused by improper operation timing. When the electrician carries out the overhaul work, the system generates a reminder, such as "under repair", prohibiting the nearby employees from turning on the power switch of the overhaul. When the overhaul work is completed, the system reminds the nearby employees to work normally.

\subsubsection{Ability assessment module}

The ability assessment has three parts. One part is the usual performance appraisal, which gives the corresponding score according to the employees' performance on daily work. The second part is the personal assessment. The system randomly selects different topics for assessment from the existing questions bank according to the needs of different types of work. It will automatically generate results, analyses and records the situation of assessment. The third part is the collaborative assessment. It pays more attention to assessing the ability of collaborative work when testing each person's ability to do things independently. Employees query scores through the examination subjects, departments, and types of work, user information or other information. The person in charge can check the examination results and analysis of all the employees in a department.

AR assessment is the part that is different from the traditional assessment in the system. After employees login into the system and select assessment mode, the system will choose appropriate problems to assess the employees according to their positions, train projects and other information. The employees need to complete the work independently within the limited time. The system assesses operations of employees and gives corresponding opinions according to the missing items. It also records these missing items as the key items and increases the frequency of appearances in the subsequent examinations. All these help employees to form the correct self-awareness, and motivate employees constantly to improve themselves. Only the missing items are corrected in training, the unsafe behavior of employees at work can be reduced. Operate correctly can reduce possible errors in the work process to achieve the purpose of safety production.

\subsubsection{Background management module}

Background management module mainly involves the following two aspects. One part is the management of various roles, such as employees, responsible persons, experts, administrators and other roles. Different users have different permissions. For example, the maintenance worker in charge of the circuit can only see the experts in the circuit. When uploading or downloading the resources, the users of different types of work can only operate the resources related to the positions. The responsible persons can import the users in batches, view different employees' learning records or assessment results, and determine its ability to be competent for the work according to the employees' performance. The second part is the daily work of the administrator, including maintain the system, update database, resource classification, expert management and other aspects of work. Its purpose is to achieve stable and orderly operation of the system.

\section{Conclusion}

The framework of mine safety training system based on augmented reality (AR) and cloud platform (CC) is put 
forward after comparing the existing training methods. Some conclusions could be drawn as following:

(1) Augmented reality technology could create a mine safety training environment where both mine theoretical knowledge and practical operation can exist simultaneously.

(2) Marker-based AR has the advantages of simple algorithm, high speed and low requirements on environmental conditions etc. It could meet the requirements of real-time and accuracy in mine training.

(3) The realization of AR training modules mainly depends on technologies such as display technology, tracking registration technology and interactive technology.

(4) The cloud platform is characterized as large scale, elasticity, and high stability. These characteristics determine that the cloud platform can be applied to mine safety training.

(5) The cloud platform can store large amounts of mine data and adjust these data according to the actual situation.

(6) This safety train framework includes several functional modules and their operation flows. These modules achieve the functions such as behavioral standardization and theoretical knowledge learning.

This system brings great convenience to mine training. It saves training costs, reduces the risks of actual operation and contributes to the formation of employees' safety awareness. It is of great significance to cultivate talents in mining enterprises and realize safe production. The safety training system could not only be applied to mines, but also could be designed into the corresponding system to meet the needs of various enterprises. For college students, the introduction of this safety training system can improve the form of teaching. It combines the theoretical knowledge with practical work, achieving the transition to work at school. For enterprises and government departments, the system can be designed into a display system. It can introduce products in a more vivid way, which not only enrich the form of work, but also save time and resources. The safety training system has broad application prospects.

\section{Acknowledgement}

This research project is made possible through the financial support from National Key R\&D Program of China (2017YFC0805100, 2016YFC0801305).

\section{References}

Cheng, Z., 2012. Research on architecture and educational application of smartphone augmented reality system. China Educational Technology, (08): 134 - 138. (in Chinese)

Gao, G.F. and F. Wang, 2016. Talking about the problems and countermeasures of mining staff training. Science \& Technology Economy Market, 150 - 151. (in Chinese)

Guo, W., 2017. The development and application of augmented reality technology. Technology Innovation and Application, (20): 180 - 182. (in Chinese)

Hong, Y.L., 2013. Coal mine accident simulation and safety training system base on virtual reality technology. Safety in Coal Mines, 44(08): 96 - 98. (in Chinese)

Li, G.R., P.F. Tian and H.B. Liu, 2014. Coal mine safety risk pre-control management cloud platform design. China Safety Science Journal, 24(02):138 - 144. (in Chinese)

Liu, F., Y.C. Ye and Y. Huang, 2007. Discussion on safety management system for metallic mine. Industrial Safety and Environmental Protection, 33(9): 58 - 61. (in Chinese)

Liu, Y., S.Q. Li and J.F. Wang, 2014. Interactive operation of physically-based slender flexible parts in an augmented reality environment. Science China Technological Sciences, 57(7): 1383 - 1391.

Liu, Y., L.F. Hu and Z.L. Tan, 2015. Research on visualization safety training of coal mine based on virtual reality. China Coal, 41(06): 79 - 83. (in Chinese)

Liu, G., L. Li. and Z.Q. Wang, et al, 2016. Research on the method about on-site training of safety production for the large petrochemical equipment based on augmented reality. Safety Health \& Environment, 16(12): 52 - 55. (in Chinese)

Ni, X.Y., J.R. Zheng and W. Zhou, 2009. Design and implementation of augmented reality software system. Computer Engineering and Design, 30(9):2297 - 2300. (in Chinese)

Sun, E.J., A Nieto, and Z.X. Li, 2010. GPS and Google Earth based 3D assisted driving system for trucks in surface mines. Mining Science and Technology, 20(1): $138-142$.

Sun, E.J., X.K. Zhang, and Z.X. Li, 2012. The internet of things (IOT) and cloud computing (CC) based tailings dam monitoring and pre-alarm system in mines. Safety Science, 50(4): 811 - 815 .

$\mathrm{Xu}$, H.L., 2017. Research on mine disaster simulation and rescue training system. China Coal, (11): 69 - 73. (in Chinese) 